\title{
Interferência do sistema de cultivo, radiação UV-C e método de secagem na qualidade da farinha de subprodutos de uva
}

\section{Interference of the cultivation system, UV-C radiation and drying method in the quality of grape by-products flour}

\author{
Leilane Demito LOPEZ1'; Ellen Porto PINTO²; Bruna Raquel BÖRGER ${ }^{3}$; Kelen Fabiana Cavalli \\ KAIPERS $^{4}$; Luciano LUCCHETTA ${ }^{5}$; Ivane Benedetti TONIAL ${ }^{6}$
}

\begin{abstract}
${ }^{1}$ Mestre em Tecnologia de Alimentos pelo Programa de Pós-Graduação em Tecnologia de Alimentos (PPGTAL) da Universidade Tecnológica Federal do Paraná (UTFPR). E-mail: leilanedl@yahoo.com.br

2 Doutora Professora/Pesquisadora do Curso de Tecnologia de Alimentos da Universidade Tecnológica Federal do Paraná (UTFPR). E-mail: ellenporto@utfpr.edu.br

${ }^{3}$ Acadêmica do Curso Superior de Tecnologia em Alimentos Universidade Tecnológica Federal do Paraná (UTFPR). E-mail: brunaraquel12@gmail.com

${ }^{4}$ Mestranda do Programa de Pós-Graduação em Tecnologia de Alimentos (PPGTAL) Universidade Tecnológica Federal do Paraná (UTFPR). E-mail: kelencavalli@hotmail.com

${ }^{5}$ Doutor Professor Permanente/Pesquisador do Programa de Pós-Graduação em Tecnologia de Alimentos (PPGTAL)

Universidade Tecnológica Federal do Paraná (UTFPR).E-mail: lucianolucchetta@gmail.com

${ }^{6}$ Autor para correspondência. Doutora Professora Permanente/Pesquisadora do Programa de Pós-Graduação em Tecnologia de Alimentos (PPGTAL. Coordenação do Curso de Tecnologia em Alimentos pela Universidade Tecnológica Federal do Paraná (UTFPR). Linha Santa Bárbara s/n CEP 85601-970 - Francisco Beltrão - PR - Brasil. Telefone: (46) 3520-2600, Ramal: 2665. E-mail: ivane@utfpr.edu.br
\end{abstract}

Recebido em: 26-03-2015; Aceito em: 25-07-2017

\section{Resumo}

Objetivou-se neste estudo investigar a influência dos sistemas de produção agrícola (orgânico e convencional), da radiação UV-C e tipo de secagem (estufa e liofilização) na composição química e físico-química de resíduos de uva e da farinha formulada a partir destes resíduos. Para isso parte das amostras de resíduos de uva Vitis labrusca, cv. 'Concord' (safra 2011/2012) foram expostas ao estresse abiótico por radiação UV-C para posterior avaliação. Para obtenção da farinha foram utilizados dois métodos de secagem: Estufa e liofilização. As amostras de resíduos e de farinha foram avaliadas quanto aos parâmetros físico-químicos (umidade, $\mathrm{pH}$, acidez, cinzas proteína e fibra bruta), compostos fenólicos, atividade antioxidante e antocianinas totais. Os resultados mostraram que para os parâmetros físico-químicos não foram observadas grandes variações de valores entre as formas de cultivo (orgânico e convencional), radiação e método de obtenção da farinha (estufa e liofilização). A liofilização proporcionou menores perdasdos níveis de compostos fenólicos. A aplicação de radiação UV-C influenciou nos teores de antocianinas e compostos fenólicos para a maioria dos tratamentos e a farinha proveniente do sistema de cultivo orgânico apresentou maior atividade antioxidante.

Palavras-chave adicionais: compostos fenólicos; estresse abiótico; produção orgânica.

\begin{abstract}
The objective of this study was to evaluate the influence of agricultural production systems (organic and conventional), UV-C radiation and type of drying (hothouse and lyophilization) on the chemical and physicochemical composition of grape residues and flour formulated at from these residues, as well as the interference of stress on these characteristics. For this, part of the grape residue samples Vitis labrusca, cv. 'Concord' (2011/2012 harvest) were exposed to abiotic stress by UV-C radiation for further evaluation. To obtain the flour were used two drying methods: hothouse and lyophilization. Samples of waste and your flours were analyzed for physico-chemical (moisture, $\mathrm{pH}$, acidity, ash, protein and fiber), phenolic compounds, antioxidant activity, and anthocyanins. The results showed that for the physical-chemical parameters doesn't were observed large variations of values between forms of cultivation (organic and convencional), radiation and method of obtaining flour (hothouse and lyophilization). However, lyophilization show lower levels of maintenance of phenolic compounds. The application of UV-C radiation influenced anthocyanin and phenolic compounds contents for most treatments, while flour from organic cultivation system showed highest antioxidant activity.
\end{abstract}

Additional keywords: abiotic stress; organic productions; phenolics compounds. 


\section{Introdução}

No cultivo da videira, fatores como temperatura, radiação solar, umidade relativa do ar e disponibilidade hídrica no solo influenciam na produtividade e na qualidade (Nilson, 2010). Assim, sistemas de cultivo, como o orgânico e o convencional, podem também interferir nos parâmetros de produtividade e de qualidade. No sistema de cultivo orgânico, não são utilizados produtos químicos sintéticos. As plantas em cultivo orgânico de produção recebem um manejo de modo a estimular os próprios mecanismos de defesa contra agentes exógenos (microorganismos, radiações, entre outros) e se adaptarem ao ecossistema (Pinto et al., 2016). O mecanismo natural de defesa das plantas é obtido por meio do metabolismo secundário, com produção de compostos, como, por exemplo, o resveratrol, dentre outros compostos fenólicos (Freitas et al., 2010). Os compostos fenólicos são substâncias aromáticas que têm ação antioxidante, atuando na estabilização de radicais livres. Seus teores estão relacionados com a espécie da planta, cultivo, maturação, origem geográfica e processo de armazenamento (Vedana et al., 2008).

A uva é considerada fonte de compostos fenólicos que interferem na coloração e no sabor, sendo que as diferentes cultivares apresenta diferentes perfis e conteúdos destes compostos (Abe et al., 2007). Os compostos fenólicos presentes nas uvas podem ser classificados em flavonoides (flavanóis, flavonóis e antocianinas) e não flavonoides (ácidos fenólicos, hidroxibenzoicos e hidroxicinâmicos) (Abe et al., 2007), além do resveratrol (polifenolestilbeno).

O resveratrol apresenta propriedades antinflamatórias, antimutagênicas, anticancerígenas, entre outras. É um dos principais componentes da uva, sendo sintetizado em resposta ao estresse abiótico, como, por exemplo, a exposição à radiação UV-C (Pan et al., 2009).

A radiação UV-C induz um estresse e afeta o metabolismo fenólico, tanto na síntese de resveratrol, como na síntese de chalcona e seus derivados, os quais atuam na inibição da agregação plaquetária, nas atividades de vasorrelaxamento e dilatação, na obesidade e em doenças degenerativas (Naderali, 2009).

Considerando os benefícios dos compostos fenólicos presentes na uva e nos seus resíduos, pesquisadores vêm estudando formas de incorporar compostos fenólicos em alimentos. É o caso de Oliveira et al. (2009), que caracterizaram a farinha de semente e a casca de uva para enriquecimento de alimentos processados, e Natividade (2010), que elaborou farinhas com resíduos de diferentes cultivares de uva provenientes da produção artesanal de suco e aplicou em iogurtes. Já Perin \& Schott (2011) elaboraram uma farinha a partir do bagaço gerado no processamento de suco de uva e desenvolveram um biscoito.

Há de se considerar também que os diferentes métodos de secagem podem influenciar na conservação das frutas bem como de seus compostos.
Assim, no intuito de verificar a influência que esta variável possui, foram utilizados dois métodos de secagem: em estufa e liofilização.

O objetivo deste estudo foi investigar a influência dos sistemas de produção agrícola (orgânico e convencional), radiação UV-C e tipo de secagem (estufa e liofilização) na composição química e físicoquímica de resíduos de uva Vitis labrusca, cv. 'Concord' e da farinha formulada a partir destes resíduos.

\section{Material e métodos}

O estudo foi conduzido com uvas Vitis labrusca, cv. Concord, provenientes de dois vinhedos comerciais localizados no município de Verê, região sudoeste do Paraná-Brasil. Esse município está localizado a uma altitude de 485 metros, latitude de $25^{\circ}=52^{\prime}$ 43 " S e longitude de $52^{\circ} 54^{\prime} 28^{\prime \prime}$ W, sob clima subtropical. As uvas foram colhidas de vinhedos de 10 anos, conduzidos em sistema latada, oriundos de cultivo orgânico e convencional, localizados em condições climáticas e edafológicas semelhantes. A colheita foi realizada quando a uva atingiu a maturação tecnológica, caracterizada pela estabilização no acúmulo de sólidos solúveis totais.

Parte das uvas utilizadas no experimento foi previamente submetida ao tratamento com radiação UV-C, segundo metodologia descrita por Cantos et al. (2000), com modificações, e a outra parte foi utilizada sem o tratamento radioativo. A taxa fluência da radiação empregada foi de $65,6 \mathrm{~J} \mathrm{~m}^{-2}$ e a distância da fonte luminosa foi de $30 \mathrm{~cm}$. Utilizaram-se três lâmpadas UV-C $(90 \mathrm{~W})$ onde as amostras permaneceram à exposição por 10 minutos. As amostras radiadas foram estocadas por três dias à temperatura ambiente $\left( \pm 25^{\circ} \mathrm{C}\right)$, na ausência de luz, para permitir a indução da biossíntese de compostos fenólicos.

O resíduo foi obtido pelo processamento do suco. Uma parte dos resíduos, compostos por cascas e sementes, foi armazenada em freezer, a $-18^{\circ} \mathrm{C}$, para avaliação de sua qualidade na forma in natura, $\mathrm{e}$ o restante foi submetido à secagem. Para a obtenção da farinha, o resíduo foi submetido a dois processos de secagem, sendo estes: a secagem em estufa e a lioflização. Para secagem em estufa, o resíduo foi submetido a $60{ }^{\circ} \mathrm{C}$, por 5 dias. Após seco e resfriado, $\mathrm{O}$ resíduo foi triturado em processador de alimentos e peneirado em peneiras de 20 mesh.

Para o processo de secagem por liofilização, utilizou-se um liofilizador da marca LioBrás, modelo LioTop L101. Depois de congeladas a $-32{ }^{\circ} \mathrm{C}$, as amostras foram submetidas a vácuo (pressão interna de aproximadamente $100 \mathrm{mmHg}$ ), durante 4 dias, para a retirada da água por sublimação. O resíduo seco foi triturado em processador de alimentos e peneirado em peneiras de 20 mesh. Ambas as farinhas foram embaladas a vácuo e armazenadas sob refrigeração até a utilização.

Para a determinação dos parâmetros físicoquímicos, os teores de umidade, $\mathrm{pH}$, acidez, cinzas, fibra bruta e proteína foram realizados de acordo com 
metodologias descritas pelo Instituto Adolfo Lutz (2008). Extratos hidroalcoólicos foram preparados para a realização das análises de compostos fenólicos, antocianinas e atividade antioxidante, tanto com o resíduo da uva, quanto com a farinha, de acordo com Vedana et al. (2008). Foram homogeneizados $60 \mathrm{~g}$ de amostra em $60 \mathrm{~mL}$ de etanol a $80 \%$, durante 10 minutos, e centrifugadas a $3.500 \mathrm{rpm}$. Separou-se o sobrenadante e manteve-se a $-18 \stackrel{\circ}{\circ}$ até o momento das análises.

A atividade antioxidante foi determinada pelo método DPPH (2,2-difenil-1-picril-hidrazila), conforme descrito por Brand-Williams et al. (1995), com modificações de Rufino et al. (2007). Os resultados foram expressos em $\mathrm{EC}_{50}$ ( $\mathrm{g}$ farinha $\mathrm{g}^{-1} \mathrm{DPPH}$ ou $\mathrm{g}$ de resíduo $\left.\mathrm{g}^{-1} \mathrm{DPPH}\right)$. A atividade sequestrante de radicais livres foi obtida a partir de uma curva-padrão de Trolox (6- hydroxy-2,5,7,8-tetramethylchromane-2-carboxylicacid). Os resultados foram expressos em TEAC $(\mu \mathrm{mol}$ de trolox $\mathrm{g}^{-1}$ farinha ou $\mu \mathrm{mol}$ de trolox $\mathrm{g}^{-1}$ resíduo).

Para a determinação dos compostos fenólicos, empregou-se a metodologia descrita por Singleton \& Rossi (1965). As antocianinas totais foram avaliadas por espectrofotometria em comprimentos de $520 \mathrm{~nm}$ e $700 \mathrm{~nm}$ segundo Fuleki \& Francis (1968).

Para a análise estatística dos dados, utilizou-se análise de variância (ANOVA) a $5 \%$ de probabilidade e teste de Tukey para comparação das médias, através do software Statistica, versão 7.0 (Statsoftlnc, 2004).

\section{Resultados e discussão}

A Tabela 1 apresenta os resultados dos parâmetros físico-químicos dos resíduos de uvas.

Tabela 1- Parâmetros físico-químicos dos resíduos de uva na forma in natura. Physicochemical parameters of grape waste in natura form.

\begin{tabular}{lcccccc}
\hline Tratamentos & \multicolumn{6}{c}{ Parâmetros } \\
\cline { 2 - 7 } & $\begin{array}{c}\text { Umidade } \\
(\%)\end{array}$ & $\mathrm{pH}$ & $\begin{array}{c}\text { Acidez } \\
(\%)\end{array}$ & $\begin{array}{c}\text { Fibra Bruta } \\
(\%)\end{array}$ & $\begin{array}{c}\text { Cinzas } \\
(\%)\end{array}$ & $\begin{array}{c}\text { Proteína } \\
(\%)\end{array}$ \\
\hline $\mathrm{CC}$ & $73,50 \pm 1,31 \mathrm{~b}$ & $4,02 \pm 0,16 \mathrm{ab}$ & $0,40 \pm 0,04 \mathrm{ab}$ & $11,26 \pm 0,76 \mathrm{a}$ & $1,16 \pm 0,09 \mathrm{a}$ & $8,95 \pm 0,02 \mathrm{c}$ \\
CO & $72,80 \pm 3,51 \mathrm{c}$ & $3,96 \pm 0,03 \mathrm{~b}$ & $0,38 \pm 0,06 \mathrm{~b}$ & $10,63 \pm 1,11 \mathrm{~b}$ & $0,92 \pm 0,16 \mathrm{c}$ & $8,90 \pm 0,72 \mathrm{~d}$ \\
CC - UV-C & $72,70 \pm 1,56 \mathrm{c}$ & $4,05 \pm 0,10 \mathrm{ab}$ & $0,41 \pm 0,04 \mathrm{a}$ & $10,30 \pm 0,17 \mathrm{~d}$ & $1,12 \pm 0,15 \mathrm{~b}$ & $9,08 \pm 0,17 \mathrm{~b}$ \\
CO - UV-C & $74,30 \pm 1,25 \mathrm{a}$ & $4,10 \pm 0,05 \mathrm{a}$ & $0,40 \pm 0,03 \mathrm{ab}$ & $10,36 \pm 0,38 \mathrm{c}$ & $0,85 \pm 0,03 \mathrm{~d}$ & $9,56 \pm 0,25 \mathrm{a}$ \\
\hline
\end{tabular}

CC: Cultivo Convencional; CO: Cultivo Orgânico; CC - UV-C: Cultivo Convencional com aplicação de UV-C; CO - UV-C: Cultivo Orgânico com aplicação de UV-C. Os resultados são médias \pm desvio-padrão. Médias em uma mesma coluna, seguidas de letras minúsculas iguais, não diferem estatisticamente $(p>0,05)$ pelo teste de Tukey.

Os parâmetros analisados apresentaram diferença $(p<0,05)$ quando se compara o tipo de cultivo e a aplicação de radiação UV-C, revelando que o resíduo de uva pode ser considerado uma importante fonte de proteína e fibra bruta.

A umidade dos resíduos de uvas apresentou valores que variaram de $72,70 \%$ (CC-UVC) a 74,30\% (CO-UVC). Estes valores podem não ser benéficos aos alimentos, uma vez que altos teores de umidade contribuem para a perecibilidade (Marques et al., 2010).

A aplicação da radiação UV-C pode ser considerada uma aliada na conservação dos frutos, pois, de acordo com Cia et al. (2009), quando estes são expostos a baixas doses de radiação UV-C, mudanças são induzidas, incluindo a produção de compostos antifúngicos e 0 atraso no amadurecimento.

A acidez do resíduo de uva apresentou-se inferior aos valores encontrados por Santos et al. (2011) em uvas de sete diferentes variedades (3,7 a 4,9\%). A acidez está diretamente associada ao estado de maturação dos frutos, parâmetro este de grande interesse para a indústria de processamento de frutos, pois está relacionada ao flavour e à qualidade tecnológica (Rizzon \& Sganzerla, 2007).

O teor de cinzas no resíduo da uva diferiu estatisticamente entre as formas de cultivo e o tratamento com UV-C. Os índices foram mais elevados nos resíduos CC, que podem estar associados, de acordo com o Instituto Brasileiro de Vinhos (2010), com a disponibilidade mineralógica do solo, irrigação, emprego de adubação, etc.

Níveis consideráveis de fibras $(10,30 \%$ para CC-UVC a $11,26 \%$ para CC) foram encontrados no resíduo de uva. Pelos altos teores de fibras revelados neste subproduto, infere-se que seu consumo pode atuar na prevenção de doenças cardiovasculares, na redução dos níveis de glicose e lipídios (Marques et al., 2010).

A fração proteica variou de $8,90 \%$ (CO) a 9,56\% (CO-UVC), sendo o terceiro maior constituinte dos parâmetros analisados.

A Tabela 2 apresenta os resultados dos principais parâmetros físico-químicos das farinhas de resíduos de uva obtidas por dois métodos de secagem: estufa e liofilização.

Os teores de água, de fibra e de cinzas não apresentaram diferença $(p>0,05)$ para o tipo de cultivo, radiação e forma de secagem do resíduo de uva. $\mathrm{O}$ teor de umidade variou de $3,71 \%$ (CO 1) a 3,88\% (COUVC 2), valores estes abaixo do limite máximo $(15,0 \%)$ estabelecido pela ANVISA para farinhas (Brasil, 2005), conferindo estabilidade frente ao desenvolvimento microbiológico e aumentando a vida de prateleira da farinha. Ferreira (2010) encontrou, em farinha de semente e casca de uva, valores superiores aos encontrados neste estudo, com média de 7,50\% e $6,80 \%$, respectivamente. 
Tabela 2- Parâmetros físico-químicos da farinha de resíduo de uva obtida por secagem em estufa (1) e liofilização (2). Physicochemical parameters of the grape residue flour obtained by drying in oven (1) and lyophilization (2).

\begin{tabular}{|c|c|c|c|c|c|c|}
\hline \multirow[b]{2}{*}{ Tratamentos } & \multicolumn{6}{|c|}{ Parâmetros } \\
\hline & $\begin{array}{c}\text { Umidade } \\
(\%)\end{array}$ & $\mathrm{pH}$ & $\begin{array}{c}\text { Acidez } \\
(\%)\end{array}$ & $\begin{array}{c}\text { Fibra Bruta } \\
(\%)\end{array}$ & $\begin{array}{c}\text { Cinzas } \\
(\%)\end{array}$ & $\begin{array}{c}\text { Proteína } \\
(\%)\end{array}$ \\
\hline CC (1) & $3,84 \pm 0,23^{\underline{a}}$ & $3,30 \pm 0,03^{c}$ & $3,50 \pm 0,10^{a}$ & $19,36 \pm 1,56^{\mathrm{a}}$ & $5,81 \pm 0,10^{\mathrm{a}}$ & $12,10 \pm 0,40^{\text {cd }}$ \\
\hline $\mathrm{CO}(1)$ & $3,71 \pm 0,19 \mathrm{a}$ & $3,50 \pm 0,09^{b}$ & $3,35 \pm 0,08^{\mathrm{ab}}$ & $19,28 \pm 1,18^{\mathrm{a}}$ & $5,84 \pm 0,37^{\underline{a}}$ & $13,37 \pm 0,24^{b}$ \\
\hline CC -ÚV-C (1) & $3,78 \pm 0,52^{\mathrm{a}}$ & $3,40 \pm 0,06^{b c}$ & $3,30 \pm 0,13^{a b}$ & $18,87 \pm 1,27^{\mathrm{a}}$ & $5,90 \pm 0,20^{\underline{a}}$ & $13,43 \pm 0,27^{b}$ \\
\hline CO -UV-C (1) & $3,83 \pm 0,30^{\mathrm{a}}$ & $3,40 \pm 0,03^{b c}$ & $3,40 \pm 0,05^{a b}$ & $19,96 \pm 0,69 \mathrm{a}$ & $5,87 \pm 0,28^{a}$ & $14,34 \pm 0,22^{\underline{a}}$ \\
\hline CC (2) & $3,79 \pm 0,12^{\mathrm{a}}$ & $3,90 \pm 0,04^{\underline{a}}$ & $3,10 \pm 0,13^{b}$ & $20,36 \pm 0,85^{\mathrm{a}}$ & $5,76 \pm 0,38^{a}$ & $12,41 \pm 0,17^{c}$ \\
\hline $\mathrm{CO}(2)$ & $3,85 \pm 0,48^{a}$ & $3,92 \pm 0,04^{a}$ & $3,13 \pm 0,16^{b}$ & $20,86 \pm 1,43^{a}$ & $6,27 \pm 0,51^{\underline{a}}$ & $12,65 \pm 0,15^{c}$ \\
\hline CC -ÚV-C (2) & $3,81 \pm 0,16^{a}$ & $3,86 \pm 0,04^{a}$ & $3,20 \pm 0,13^{a b}$ & $21,30 \pm 0,55^{\mathrm{a}}$ & $6,17 \pm 0,35^{\mathrm{a}}$ & $11,55 \pm 0,23^{d}$ \\
\hline CO -UV-C (2) & $3,88 \pm 0,07^{a}$ & $3,82 \pm 0,13^{\underline{a}}$ & $3,15 \pm 0,11^{b}$ & $19,98 \pm 0,38^{a}$ & $6,21 \pm 0,51^{a}$ & $12,41 \pm 0,25^{c}$ \\
\hline
\end{tabular}

CC: Cultivo Convencional; CO: Cultivo Orgânico; CC - UV-C: Cultivo Convencional com aplicação de UV-C; CO- UV-C: Cultivo Orgânico com aplicação de UV-C. Os resultados são médias \pm desvio-padrão. Médias em uma mesma coluna, seguidas de letras minúsculas iguais, não diferem estatisticamente $(p>0,05)$ pelo teste de Tukey.

O conteúdo de proteína variou de $11,55 \%$ (CC - UVC 2) a 14,34\% (CO-UVC1), com diferença $(p<0,05)$ entre as médias. Estes valores estão próximos do encontrado por Ferreira (2010) também em estudo realizado com farinha de resíduo de uva $(14,65 \%)$. Este autor afirma que o teor de proteína da uva é dependente da cultivar, e as proteínas fazem-se presentes, principalmente na polpa da uva.

O percentual de cinzas apresentou variações de $5,76 \%$ (CC 2) a 6,27\% (CO 2). Os teores de cinzas indicam que o produto elaborado representa boa fonte de minerais (Cecchi, 2003).

Os valores de $\mathrm{pH}$ e de acidez para as farinhas provenientes da secagem em estufa variaram de 3,30 a 3,50, enquanto as farinhas obtidas pela secagem dos resíduos de uva por liofilização apresentaram valores que variaram de 3,82 a 3,92 para $\mathrm{pH}$ e 3,10 a 3,20 para acidez.

$\mathrm{O}$ valor de $\mathrm{pH}$ ideal, de acordo com Carvalho (2010), para farinhas é inferior a 4,5, valor este que delimita o desenvolvimento de microrganismos deteriorantes. Desta forma, pode-se afirmar que as farinhas analisadas se encontram com valores de $\mathrm{pH}$ que podem conferir maior estabilidade e vida de prateleira (Aquino et al., 2010). A acidez da farinha, por sua vez, está relacionada à presença de ácidos orgânicos provenientes dos resíduos da uva, e estes influenciam no sabor, odor, cor, na estabilidade e na manutenção de qualidade (Cecchi, 2003).

Dos parâmetros analisados, as fibras apresentaram-se de forma majoritária quando relacionadas aos demais componentes, com valores que variaram de $18,87 \%$ (CC-UVC 1) a $21,30 \%$ (CC-UVC 2), sendo inferiores ao verificado por Bampi et al. (2010) em farinha de uva-do-japão $(25,62 \%)$, indicando que o produto elaborado a partir de resíduo de uva (farinha) pode ser incluído na dieta como fonte de fibras.

O conteúdo de compostos fenólicos totais e de antocianinas totais presentes nos resíduos e as farinhas de resíduos de uva são mostrados na Figura 1.

Efeito positivo no acúmulo de compostos fenólicos induzidos pela exposição ao UV-C, em frutas tropicais, foi relatado por Alothman et al. (2009); no entanto, isso não foi observado no presente estudo com uvas 'Concord', as quais não revelaram influência significativa nos índices de compostos fenólicos.

Os teores de compostos fenólicos dos resíduos de uva variaram entre 257,2 $\mathrm{mg} \mathrm{GAEg}^{-1}$ (orgânico UVC) e $339,78 \mathrm{mg} \mathrm{GAEg}^{-1}$ de resíduo (convencional UVC) e apresentaram-se similares nos sistemas convencional e orgânico (Figura 1A). Fatores como variedades, região, safra, condições climáticas e métodos de extração podem influenciar nos resultados dos teores de compostos fenólicos, bem como na expressão da atividade antioxidante (Rockenbach et al., 2008). Assim, observou-se diferença $(p<0,05)$ nos teores de compostos fenólicos entre os sistemas de produção convencional e orgânico quando tratados com radiação UV-C, sendo que o resíduo de uva orgânica apresentou menor concentração destes compostos. Em estudo realizado por Pinto et al. (2016), verificaram-se diferenças entre as uvas dos dois sistemas de produção. Após aplicação da radiação UV-C, observou-se que as uvas provenientes do cultivo convencional apresentaram maiores conteúdos de compostos fenólicos.

Os teores de antocianinas nos resíduos de uva variaram de $32,95 \mathrm{mg}$ de cianidina-3-glicosídeo por $100 \mathrm{~g}$ (orgânico UVC) a 72,71 mg de cianidina-3-glicosídeo por $100 \mathrm{~g}$ de resíduo, sendo superior no resíduo de uvas de sistema convencional (Figura 1B).Os resíduos provenientes de uvas radiadas apresentaram valores menores quando comparados com as amostras não submetidas à radiação UV-C. Este fato pode ser explicado pela fotodegradação das antocianinas causada pela exposição à radiação (Pala \& Toklucu, 2013).

Os métodos de secagem dos resíduos: estufa (1) e liofilização (2) influenciaram nos teores de compostos fenólicos totais das farinhas (Figura 1C). A farinha obtida por meio do processo de liofilização apresentou teores de compostos fenólicos superiores à farinha obtida por secagem em estufa, com exceção da farinha de uva orgânica UV-C (2). O sistema de secagem por estufa resultou em maiores perdas que a liofilização, tendência esta já esperada, o que pode 
estar relacionado à menor agressão do material quando utilizada a liofilização.

A farinha de uva convencional (2) apresentou concentração de compostos fenólicos superiores quando comparada às demais farinhas. A radiação UV-C não resultou em acúmulo destes compostos e,
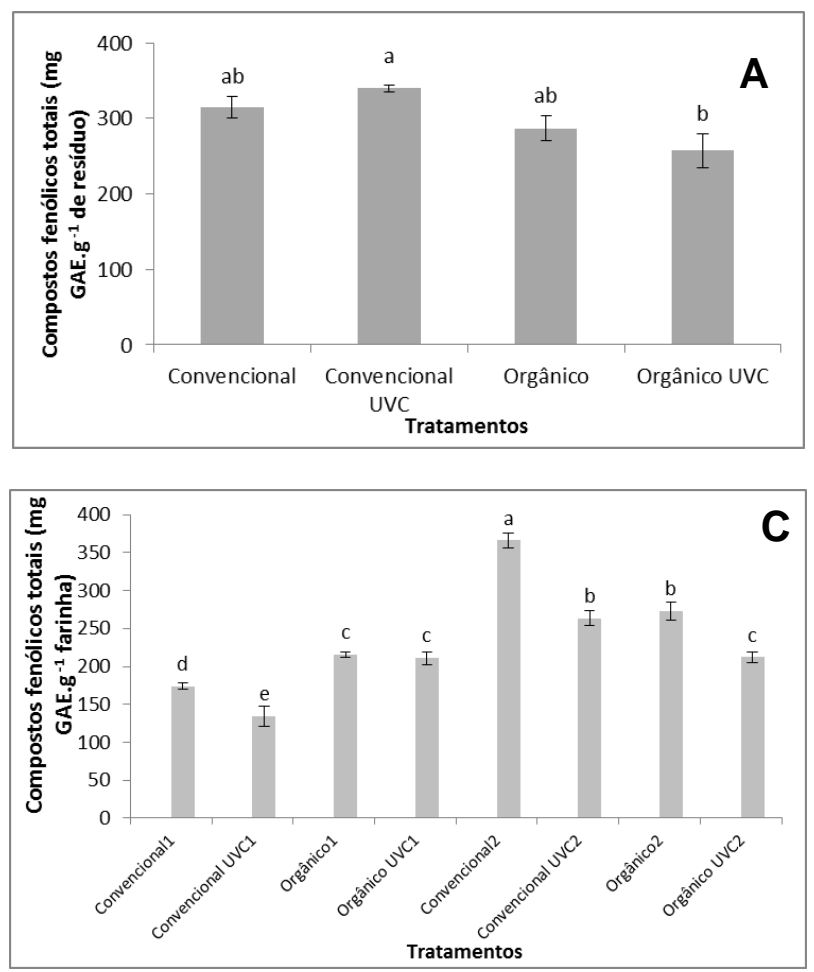

sim, reduziu sua quantidade, ocasionada, possivelmente, pelo fato de o tratamento radioativo ter sido aplicado nas frutas já maduras; no entanto, este comportamento não foi observado nos resíduos, ou seja, as etapas de preparo da farinha podem ter influenciado na diminuição dos compostos fenólicos.
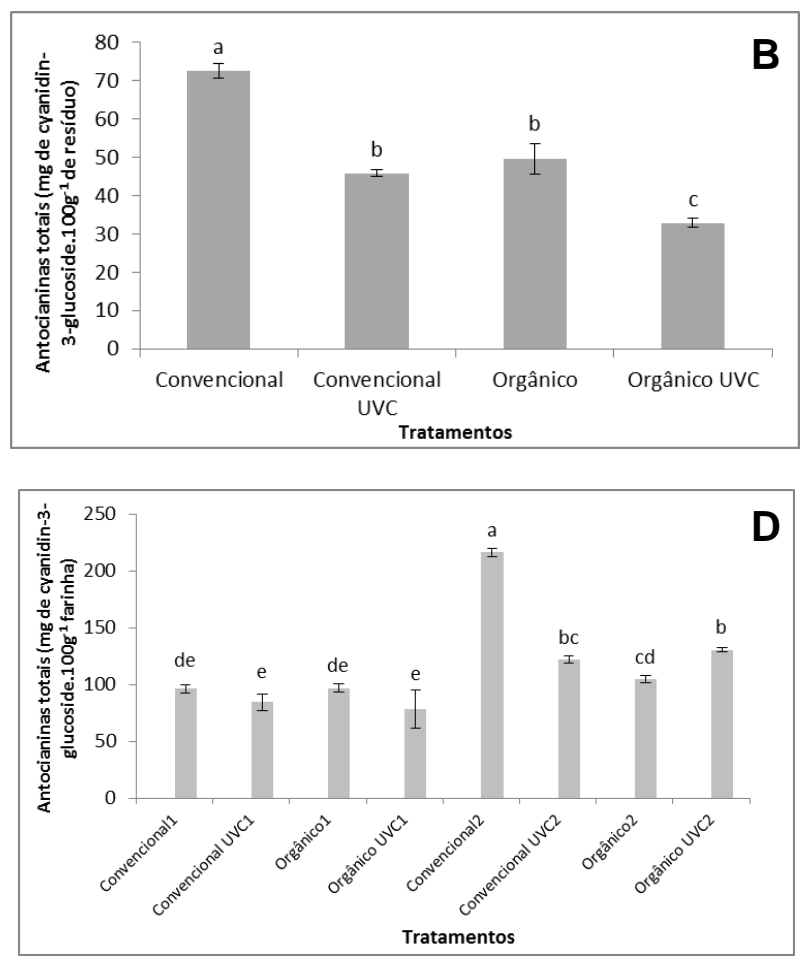

Figura 1. Compostos fenólicos totais e Antocianinas totais em resíduos (A e B) e farinhas (C e D) de uva orgânica e convencional, com e sem radiação UV-C, sendo 1 (secagem em estufa) e 2 (secagem em liofilizador).Total phenolic compounds and anthocyanins total in waste ( $A$ and $B$ ) and grape flour $(C$ and $D)$ of organic and conventional farming systems, with and without UV-C radiation, being 1 (oven drying) and 2 (lyophilization).

Quanto aos teores de antocianinas totais, houve diferença significativa, principalmente entre os métodos de secagem (Figura 1D). Os valores para as farinhas obtidas por liofilização foram superiores, destacando-se a farinha de uva convencional (2). A secagem em estufa utilizou a temperatura de $60^{\circ} \mathrm{C}$, por 5 dias, o que afetou significativamente o conteúdo de antocianinas. Estes compostos foram perdidos pelo fato de serem sensíveis à temperatura. A secagem em estufa não consegue reter tantos componentes quanto a liofilização, que é um método mais brando.

Com relação ao sistema de cultivo, as farinhas de uva orgânica (2) e convencional (2) apresentaram diferença significativa $(\mathrm{p}<0,05)$ no teor de antocianinas totais, onde a convencional (2) revelou maiores quantidades destes compostos (216,08 mg de cianidina-3glicosídeo.100 $\mathrm{g}^{-1}$ de farinha). A radiação com UV-C não influenciou nos teores de antocianinas totais das farinhas obtidas na secagem em estufa. Estes compostos também não apresentaram alterações em estudo conduzido por Pala \& Toklucu (2013) em suco de uvas submetido ao tratamento com UV-C.
Os resultados da atividade antioxidante avaliada no resíduo e nas farinhas são apresentados na Figura 2.

Os resíduos de uva orgânica apresentaram maior atividade antioxidante quando comparados aos convencionais (Figuras 2A e 2B). Os resultados observados neste estudo corroboram os de Mulero et al. (2010), que também observaram maior atividade antioxidante em uvas de sistema de produção orgânica.

Alothman et al. (2009), ao estudarem o efeito da radiação UV-C sobre a atividade antioxidante de frutas tropicais recém-colhidas, verificaram aumento da atividade antioxidante após a aplicação desta radiação. Da mesma forma, Mulero et al. (2010) observaram aumento da atividade antioxidante em uvas de cultivo orgânico e convencional após radiação. Neste estudo, porém, este aumento não foi observado para os resíduos de uva, em ambos os sistemas de cultivo.

Comparando o sistema de cultivo, a farinha de uva orgânica (2) apresentou a maior atividade antioxidante expressa em EC 50 (Figura $2 \mathrm{C}$ ). Foram necessários 201,92 g de farinha para reduzir um grama do radical DPPH. 

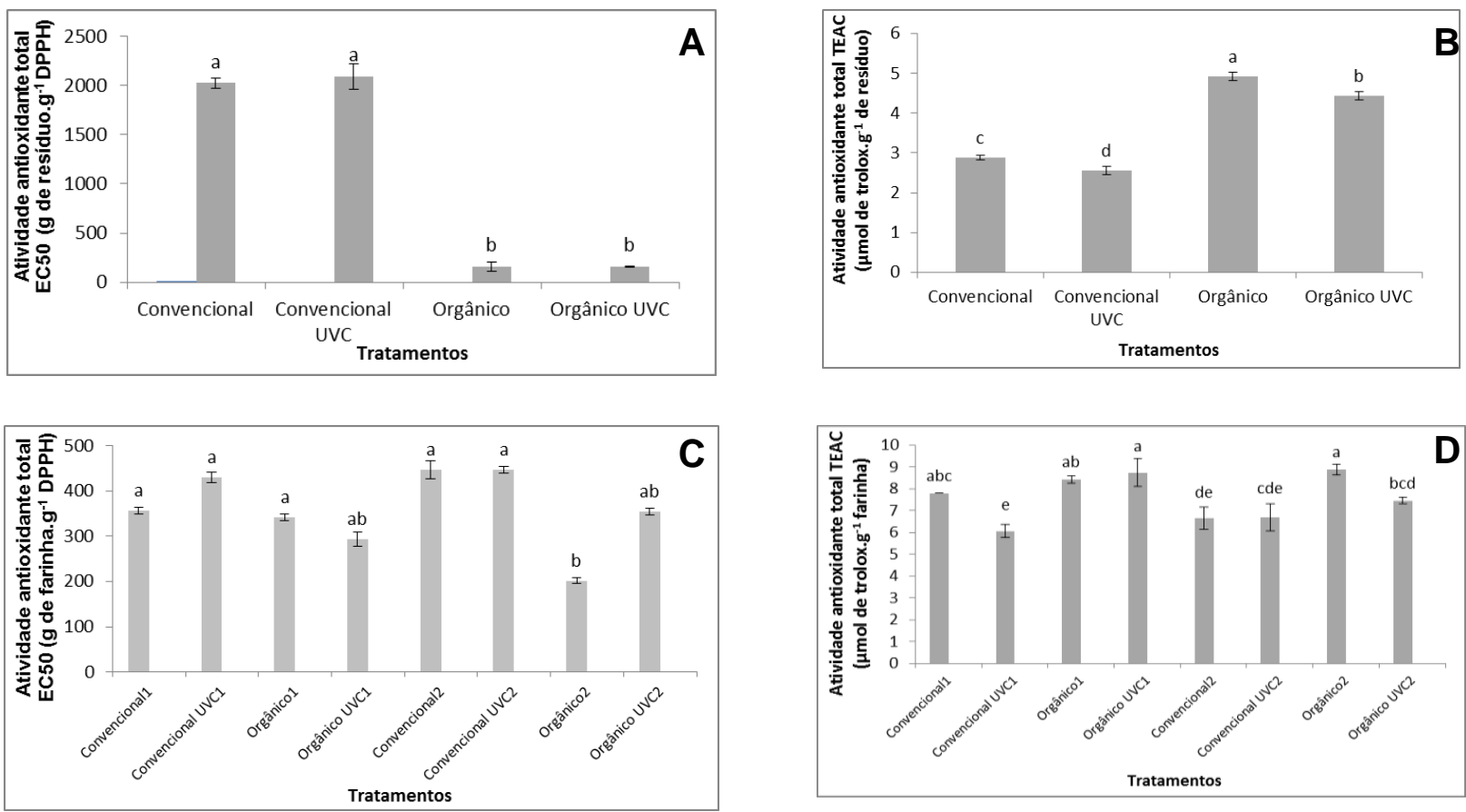

Figura 2. Atividade antioxidante $E_{50}$ e TEAC de resíduos (A e B) e farinha de resíduos (C e D) de uva de sistemas de cultivo orgânico e convencional, com e sem tratamento com radiação UV-C, sendo 1 (secagem em estufa) e 2 (secagem em liofilizador). Antioxidant Activity $E C_{50}$ and TEAC of waste ( $A$ and $B$ ) and residue flour ( $C$ and $D$ ) of grape of organic and conventional farming systems, with and without UV-C radiation, being 1 (oven drying) and 2 (lyophilization).

Quanto à interferência do tipo de secagem na atividade antioxidante expressa em TEAC (Figura 2D), destacam-se as diferenças significativas entre os tratamentos: convencional (1) e (2); orgânico UVC1 e UVC2. Em relação ao tipo de cultivo, a farinha orgânica UVC1 apresentou maior atividade antioxidante em relação à farinha convencional UVC1, assim como a orgânica (2) foi maior que a convencional (2). A aplicação da radiação UV-C reduziu a atividade antioxidante das farinhas: convencional (1) e orgânica (2).

A variação dos conteúdos de antocianinas totais revelou a existência de uma relação com os níveis de compostos fenólicos. Por outro lado, o estudo mostrou que a redução ou o aumento do conteúdo dos compostos fenólicos não necessariamente resulta em variação da atividade antioxidante. Resultados semeIhantes, em que o conteúdo de fenóis totais sofreu redução significativa sem alteração da atividade antioxidante, já foram descritos para suco de maçã (Caminiti et al., 2011). A não correlação entre atividade antioxidante e fenólicos totais está relacionada à grande variedade de compostos com atividade antioxidante, além dos fenólicos, como vitamina C, carotenoides, etc, como já descrito em estudo com outras espécies de frutas (Gil et al., 2002).

\section{Conclusões}

Os sistemas de cultivo, radiação UV-C e métodos de secagem não interferiram nos teores de fibra e cinzas das farinhas e na umidade. No entanto, afetaram o percentual de proteína.

$\mathrm{O}$ tratamento com radiação UV-C teve efeito negativo no conteúdo de compostos fenólicos e antocianinas totais na maior parte dos tratamentos, e a liofilização proporcionou menores perdas dos níveis de compostos fenólicos.

O sistema de cultivo orgânico resultou em maior atividade antioxidante comparado ao cultivo convencional, mesmo com a variação dos níveis de compostos fenólicos e antocianinas.

\section{Agradecimentos}

Os autores agradecem ao Conselho Nacional de Desenvolvimento Científico e Tecnológico (CNPqBrasil) e ao Programa de Apoio para publicação da Universidade Tecnológica Federal do Paraná (UTFPR) - Francisco Beltrão.

\section{Referências}

Abe LT, Mota RV, Lajolo FM, Genovese ML (2007) Compostos fenólicos e capacidade antioxidante de cultivares de uvas Vitis labrusca L. e Vitis vinífera L, Ciência e Tecnologia de Alimentos 27(2):394-400.

Alothman M, Bhat R, Karim AA (2009) UV radiationinduced changes of antioxidant capacity of fresh-cut tropical fruits. Innovative Food Science and Emerging Technologies 10:512-516. 
Aquino ACMS, Móes RS, Leão KMM, Figueiredo AVD, Castro AA (2010) Avaliação físico-química e aceitação sensorial de biscoitos tipo cookies elaborados com farinha de resíduos de acerola. Revista Instituto Adolfo Lutz, 69(3): 379-386.

Bampi M, Bicudo MOP, Fontoura PSG, Ribani RH (2010) Composição centesimal do fruto, extrato concentrado e da farinha de uva-do-japão. Ciência Rural 40 (11): 2361-2367.

Brand-Wiliams W, Cuvelier ME, Berset C (1995) Use of a free radical method to evaluate antioxidant activity. Food Science Technology 28, 25-30.

Brasil, Agência Nacional de Vigilância Sanitária (ANVISA). Regulamento Técnico para Produtos de Cereais, Amidos, Farinha e Farelos, Resolução RDC no 263, 22 de setembro de 2005.

Caminiti IM, Noci F, Munoz A, Whyte P, Morgan DJ, Cronin DA, Lyng JG (2011) Impact of selected combinations of non-thermal processing technologies on the quality of an apple and cranberry juice blend. Food Chemistry124 (4):1387-1392.

Cantos E, García-Viguera C, Pascual TS, TomásBarberán FA (2000) Effect of postharvest ultraviolet irradiation on resveratrol and other phenolics of $\mathrm{CV}$. Napole on table grapes. Journal Agricultural Food Chemistry 48(10):4606-4612.

Carvalho, IT (2010) Microbiologia dos alimentos. Recife: EDUFRPE,86 p.

Cecchi, HM (2003) Fundamentos teóricos e práticos em análise de alimentos. 2.ed. Campinas: Unicamp, 207p.

Cia P, Benato EA, Valentini SRT, Anjos VDA, Ponzo FS, Sanches J, Terra MM (2009) Radiação ultravioleta no controle pós-colheita de Colletotrichumgloeosporioidesem uva niagara rosada. Bragantia 68 (4):10091015.

Freitas AA, Detoni AM, Clemente E, Oliveira CC (2010) Determinação de resveratrol e características químicas em sucos de uvas produzidas em sistemas orgânico e convencional. Revista Ceres 57 (1): 001-005.

Ferreira LFD (2010) Obtenção e caracterização de farinha de bagaço de uva e sua utilização em cereais matinais expandidos. Universidade Federal de Viçosa (Tese de doutorado em Ciência e Tecnologia de Alimentos).

Fuleki T, Francis FJ (1968) Quantitative determination of anthocyanins. Determination of total anthocyanins and degradation index for cranberry juice. Journal Food Science 33 (1):78-83.
Gil MI, Tomás-Barberán FA, Hess-Pierce B, Kader AA (2002). Antioxidant capacities, phenolic compounds, carotenoids, and vitamin C contents of nectarine, peach, and plum cultivars from California. Journal of Agricultural Food Chemistry, 50, 4976-4982.

Instituto Adolfo Lutz (2008) Normas Analíticas do Instituto Adolfo Lutz: Métodos Químicos para Análise de Alimentos. 4.ed.

Instituto Brasileiro do Vinhos/ IBRAVIN. A vitivinicultura Brasileira, 2010. Disponível em: http://www.ibravin.org.br/. Acesso em 20 maio. 2017.

Marques A, Chicaybam G, Araujo MT, Manhães LRT, Sabaa-Srur AUO (2010) Composição centesimal e de minerais de casca e polpa de manga (Mangifera indica L.) cv Tommy Atkins. Revista Brasileira de Fruticultura 32 (4):1206-1210.

Mulero J, Pardo F, Zafrilla P (2010) Antioxidant activity and phenolic composite on of organic and conventional grapes and wines. Journal of Food Composition and Analysis 23 (6):569-574.

Naderali EK (2009) Obesity and cardiovascular dysfunction: a role for reveratrol? Obes. Diabetes Research Clinical Practice 3 (1):45-52.

Natividade MMP (2010). Desenvolvimento, caracterização e aplicação tecnológica de farinhas elaboradas com resíduos da produção de suco de uva. Dissertação, Pós-graduação em Ciência dos Alimentos. Universidade Federal de Lavras, Lavras-MG, 203p.

Nilson TS (2010) Influência do clima sobre os estádios fenológicos da videira e sobre a qualidade e quantidade da produção. Instituto Federal de Educação (Trabalho de conclusão de Curso Superior em Ciência e Tecnologia do Rio Grande do Sul).

Oliveira LT, Velos JCR, Teranortiz GP (2009). Caracterização físico-quimica da farinha de semente e casca de uva. II Semana de Ciência e Tecnologia do IFMG campus Bambuí e II Jornada Científica.

Pala U, Toklucu K (2013) Effects of UV-C Light Processing on Some Quality Characteristics of Grape Juices. Food Bioprocess Technology 6 (3): 719-725.

Pan QH, Wang L, Li JM (2009) Amounts and subcellular localization odstilbene synthase in response of grape berries to UV irradiation. Plant Science176 (3): 360-366.

Perin EC, Schott IS (2011). Utilização de farinha extraída de resíduos de uva na elaboração de biscoito tipo cookie. Trabalho de Conclusão de Curso. Universidade tecnológica Federal do Paraná, 61p.

Pinto EP, Perin EC, Schott IB, Da Silva Rodrigues R, Lucchetta L, Manfroi V, Rombaldi CV (2016) The effect of postharvest application of UV-C radiation on the phenolic compounds of conventional and organic grapes (Vitis labrusca cv. Concord). Postharvest Biology and Technology, v.120, p.84 - 91. 
Rizzon LA, Sganzerla VMA (2007) Ácidos tartáricos e málico no mosto de uva em Bento Gonçalves-RS. Ciência Rural 37 (3):911-914.

Rockenbach SGL, Rodrigues E, Kuskosk EM, Fett R (2008) Influência do solvente no conteúdo total de polifenóis, antocianinas e atividade antioxidante de extratos de bagaço de uva (Vitis vinifera) variedades Tannate Ancelota. Ciência e Tecnologia de Alimentos 28 (supl.): 238-244.

Rufino MSM, Alves RE, Brito ES, Morais SM, Sampaio CG, Sanchez-Alonso I, Jiménez-Escrig A, Saura-Calixto F, Borderías AJ (2007) Antioxidant protectionof White Grape pomace on restructured fish products during frozen torage. Lebensmittel Wissenscharft and Tecnology 42 (1):42-50.
Santos EHB, Azevedo LC, Batista FPR, Matos LP, Lima MS (2011) Caracterização química e sensorial de uvas desidratadas, produzidas no Vale do São Francisco para infusão. Revista Seminário de Visum 1 (2):134-147.

Singleton VL, Rossi JA (1965) Colorimetry of total phenolics with phosphomolybdic-phosphotungstic acid reagents. American Journal of Enology and Viticulture16 (3)144-153.

Statsoft Inc (2004) Statistica data analysis system version 7.0. Tulsa: Statsoft Inc.

Vedana MIS, Ziemer C, Miguel OG, Portella AC, Candido LMB (2008) Efeito do processamento na atividade antioxidante da uva. Alimentos e Nutrição 19 (2):159-165. 